\title{
Family Doctors and Lower Diagnostic Imaging Costs: How Do We Get There from Here?
}

\section{Médecins de famille et coûts d'imagerie diagnostique moins élevés : comment peut-on y arriver?}

HEATHER DAWSON, MHSC, CHE

Director, Analysis and Reporting, Health Council of Canada

$\mathrm{M}$

oira Stewart and colleagues present a promising new analysis of their data indicating that patient-centred care may contribute to lower diagnostic imaging costs, at least according to available data on practice patterns and costs in Ontario. If this positive outcome holds true, the question then is: what needs to be done to ensure that more of Canada's family doctors are able and encouraged to practise patientcentred care?

The Health Council of Canada's report, Decisions, Decisions: Family Doctors as Gatekeepers to Prescription Drugs and Diagnostic Imaging in Canada (September 2010), was intended to open the door to discussion and debate about the increasingly important but under-recognized role of family physicians as gatekeepers to pharmaceutical drugs and diagnostic imaging, two drivers of increased healthcare spending. Although we did not examine practice patterns and costs through the lens of patient-centredness of care, we can certainly agree with the authors' recommendations (and make similar recommendations ourselves) that better data and more research are needed to understand the relationships between approaches to care, compensation or incentives, system utilization, patient outcomes and costs. 
Although we did not set out to reach conclusions related to patient-centred care, based on our findings, we would suggest that it is not patient-centred care in itself that results in higher costs. Rather, owing to the complex environment within which family physicians play their gatekeeping role, there are impediments to providing patient-centred care to the fullest extent, and it is these impediments that are contributing to rising costs.

Stewart and her colleagues note that use of diagnostic testing has increased since 2000. Indeed, the most recent data show that between 1990 and 2009, the number of CT scanners in Canada more than doubled, while MRI scanners increased more than tenfold. Accordingly, in 2009, Canadians received more than four million CT exams and nearly 1.4 million MRI exams - a 58\% increase in CT exams and a 100\% increase in MRI exams compared to 2003 (Health Council of Canada 2010). Higher costs related to diagnostic imaging over the past 10 years have been directly linked to increased access to scans throughout Canada. As costs and access have increased, concerns have been raised regarding overuse and inappropriate ordering. According to the Canadian Association of Radiologists (2009), as many as 30\% of CT scans and other imaging procedures are inappropriate or contribute no useful information. While Stewart and colleagues acknowledge that they did not factor appropriateness into their analysis, the Health Council feels that appropriateness is a key consideration that must be factored into discussions associated with physicians' practice and costs, and impact on patient-centred care.

There is no doubt that for family doctors, their patients' health and safety are the primary focus of decision-making. We know that the factors that family physicians take into consideration when making treatment recommendations - a decision to order a diagnostic test, prescribe a drug, refer to a specialist or follow another course of action - are numerous and complex. They include the physician's initial medical training and efforts to stay on top of current research, the availability of new technologies, the desire to meet patients' expectations and the doctor's overall clinical judgment. We expect that models of physician compensation, including performance incentives, also play a role.

So why are physicians ordering unnecessary tests? And how does this practice relate to patient-centred care?

Based on our review of the literature and expert advice from physician and radiologist leaders in Canada, we found that inappropriate ordering is a consequence of pressures put on referring physicians by patients and by an ever-increasing workload. Physicians are ordering tests in an environment that is rapidly changing. Standards for best practice for diagnostic imaging are constantly being updated, and decision support tools to aid family physicians are limited or not easily accessible. We also found that liability and malpractice concerns may drive physicians to order more tests than needed. This environment is not conducive to patient-centred care.

In addition to the complex decision-making environment, physicians are making decisions in a practice environment that is different from 10 and even five years ago. Canada's move to primary care teams and alternative funding models that would ideally allow physicians to fully explore and take patients' desires, beliefs and capabilities into consideration - important elements 
of patient-centred care. Currently, however, we are quite limited in terms of data or evidence to show where we are in terms of the impact of primary care reform on patient-centred care.

According to the 2007 National Physician Survey, Canadian family physicians spend roughly two-thirds of their time on direct patient care. The remainder is spent on managing their practice, participating in research projects, teaching and continuing medical education. These multiple demands are squeezing the amount of time doctors spend with patients, which one Ontario study found to be roughly 10 to 15 minutes per visit (Russell et al. 2009). These time pressures are not conducive to patient-centred care.

Patient-centred care may indeed be associated with lower diagnostic costs, but until family physicians are able to practice in environments that optimize this type of care, we will not be able to measure the true impact on outcomes and costs at the system level, let alone for specific procedures.

\section{REFERENCES}

Canadian Association of Radiologists. 2009. Do You Need That Scan? Retrieved April 12, 2011. <http://www.car. ca/uploads/patient\%20info/car_cat_scan_eng.pdf>.

Health Council of Canada. 2010 (September). Decisions, Decisions: Family Doctors as Gatekeepers to Prescription Drugs and Diagnostic Imaging in Canada. Retrieved April 12, 2011. <http://healthcouncilcanada.ca/docs/ rpts/2010/HSU/DecisionsHSU_Sept2010.pdf>.

Russell, G.M., S. Dahrouge, W. Hogg, R. Geneau, L. Muldoon and M. Tuna. 2009. “Managing Chronic Disease in Ontario Primary Care: The Impact of Organizational Factors.” Annals of Family Medicine 7(4): 309-18.

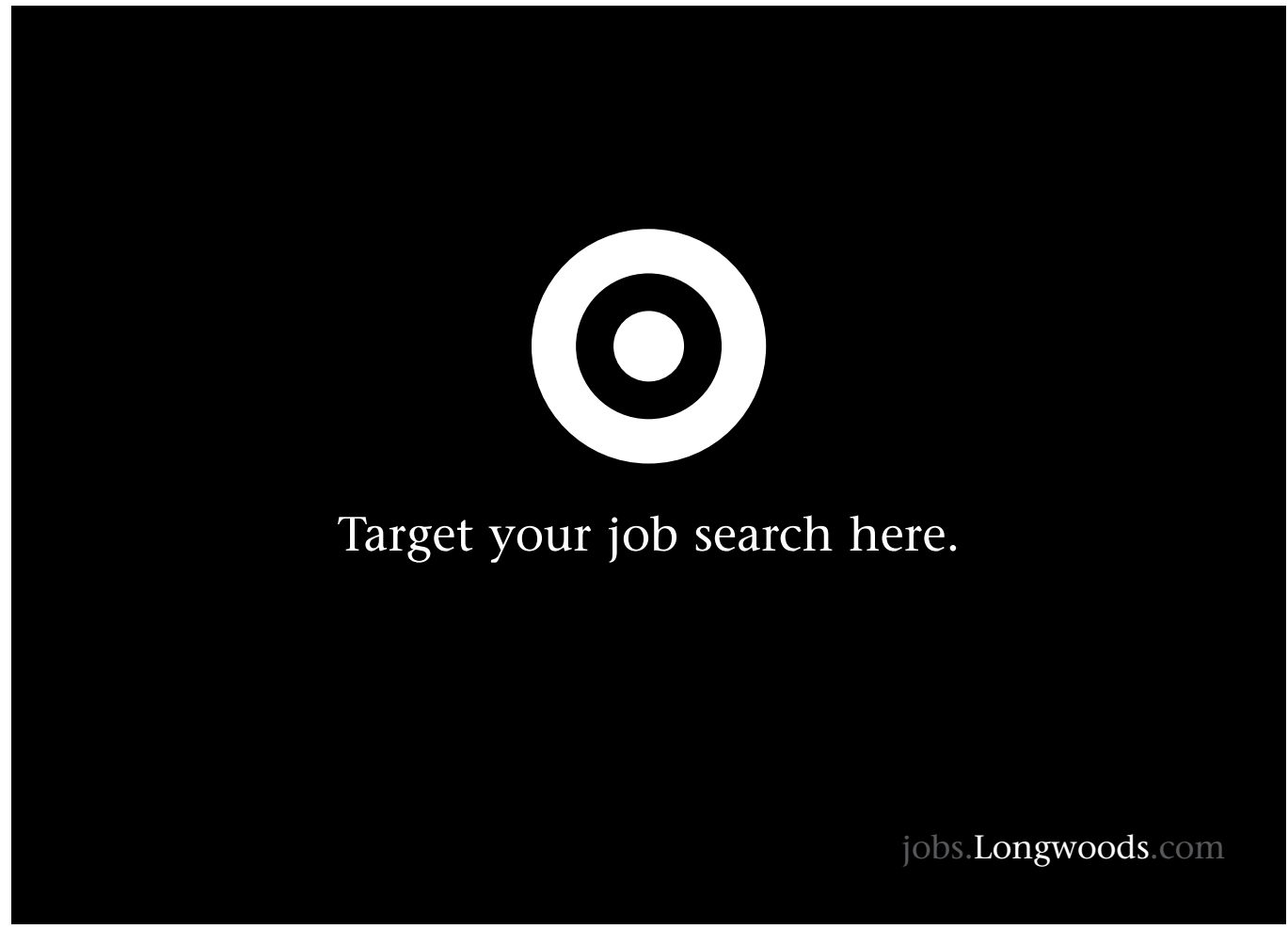

